\title{
L'accès des femmes aux livres et aux bibliothèques familiales en Gaule romaine
}

\section{Robert Bedon}

\section{(2) OpenEdition}

1 Journals

Édition électronique

URL : http://journals.openedition.org/rbnu/1158

DOI : $10.4000 /$ rbnu. 1158

ISSN : 2679-6104

\section{Éditeur}

Bibliothèque nationale et universitaire de Strasbourg

\section{Édition imprimée}

Date de publication : 1 novembre 2016

Pagination : 26-35

ISBN : 9782859230630

ISSN : 2109-2761

\section{Référence électronique}

Robert Bedon, «L'accès des femmes aux livres et aux bibliothèques familiales en Gaule romaine », La Revue de la BNU [En ligne], 14 | 2016, mis en ligne le 01 janvier 2020, consulté le 11 décembre 2020. URL : http://journals.openedition.org/rbnu/1158; DOI : https://doi.org/10.4000/rbnu.1158

\section{(c) (i) (2)}

La Revue de la BNU est mise à disposition selon les termes de la Licence Creative Commons Attribution - Pas d'Utilisation Commerciale - Partage dans les Mêmes Conditions 4.0 International. 


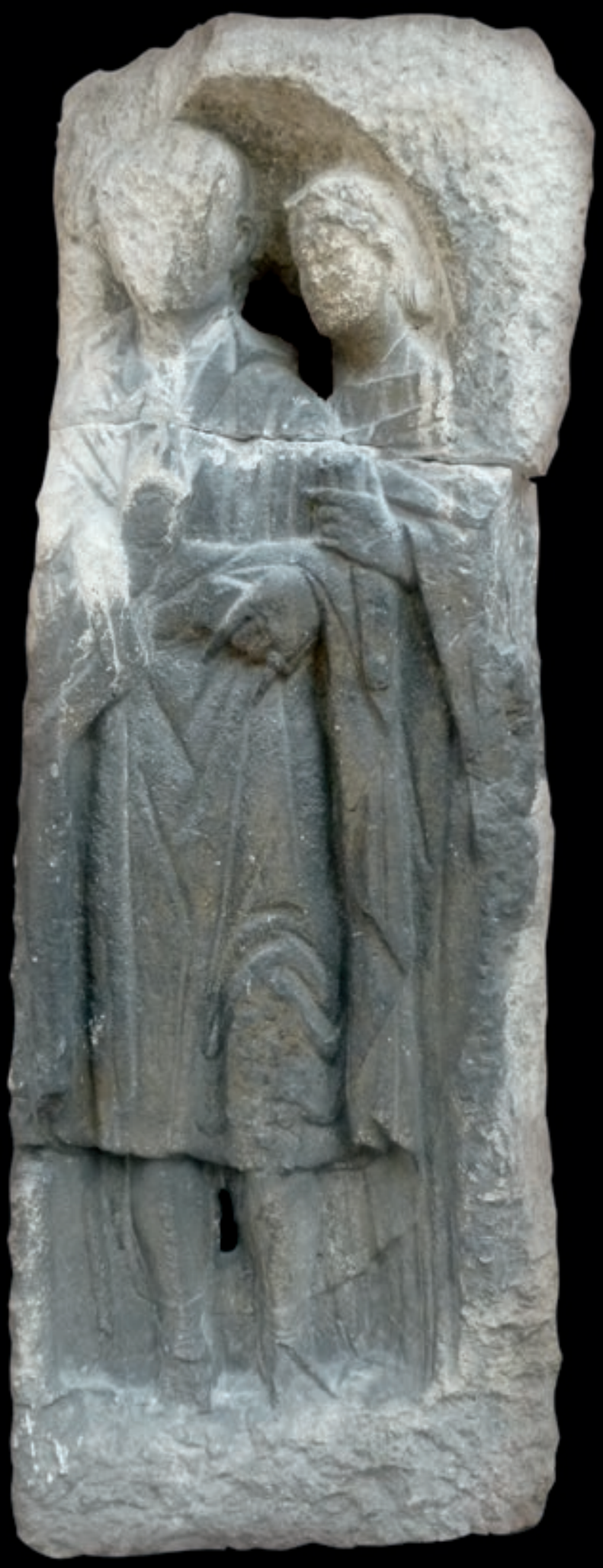

Monument funéraire d'Avaricum figurant un couple qui tient des tablettes et un volumen de luxe (collections des musées de la Ville de Bourges, Musée du Berry) 


\section{L'ACGÈS DES FEMMES AUX LIVRES ET AUX BIBLIOTHĖQUES FAMILIALES EN GAULE ROMAINE}

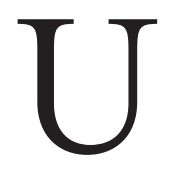

$\mathrm{n}$ regard d'ensemble sur la pratique de la lecture et la fréquentation des bibliothèques dans le monde romain antique fait apparaître essentiellement des utilisateurs masculins, évoqués par des auteurs tels que Pline le Jeune, Suétone, Fronton, Aulu-Gelle ou encore Athénée. Or quelques documents écrits et iconographiques témoignent, explicitement ou indirectement, d'une pratique féminine de la lecture dans le monde romain. Elle se localisait assez souvent dans des bibliothèques familiales, et était rendue possible par le fait que dans les familles et les milieux qui possédaient des livres, la plupart des femmes avaient appris à lire durant leur enfance, à la maison ou dans une école, ce qui leur donnait au moins un niveau d'instruction de base, mais parfois plus élevé. Le hasard de la transmission à travers les siècles de textes, d'inscriptions et de monuments funéraires porteurs de sculptures a fait que pour la Gaule romaine, cette pratique et cette fréquentation se trouvent à plusieurs reprises mentionnées, évoquées ou suggérées, en quantité suffisante pour permettre, à partir d'un choix ne retenant que les plus explicites et les plus représentatifs, de nous constituer une idée générale assez précise de cet aspect de la civilisation gallo-romaine.

\section{Des sources variées}

Le témoignage le plus détaillé nous a été transmis par un auteur de l'Antiquité tardive, Sidoine Apollinaire, dont la vie se situe entre 430 et 486 . Dans le recueil de ses lettres qu'il avait constitué, en figure une où il évoque une série d'après-midi passés dans une ambiance familiale, à l'intérieur d'un salon dédié aux livres et appartenant à la résidence rurale d'un de ses amis, située en Provence, à proximité du Gardon, dans le voisinage d'Alès. Voici le début des lignes qui nous intéressent:
"Ici des ouvrages étaient mis en grand nombre à disposition. Tu aurais cru voir des rayonnages de grammairiens, les gradins de l'Athénée ou les hautes armoires des libraires. Ils étaient toutefois rangés de telle façon que dans les livres situés près des fauteuils prévus pour les dames..." ${ }^{1}$.

D'autres indices d'une pratique féminine de la lecture, et par delà, d'une fréquentation de bibliothèques familiales, proviennent de monuments funéraires que l'on nomme des stèles, portant une représentation sculptée de défuntes. Le plus bel exemple consiste en un relief issu d'une nécropole d'Avaricum, de nos jours Bourges (voir ill. ci-contre $)^{2}$. D'une facture très soignée, mais ayant subi d'importants dommages, il montre un couple d'allure distinguée, sculpté en pied dans des dimensions proches de la réalité. L'homme en occupe le centre et la gauche. Derrière lui, dans la partie droite, se tient son épouse, la tête légèrement inclinée dans sa direction. Elle pose sa main droite sur l'épaule de son mari, et l'autre sur l'avant-bras gauche de celui-ci, simultanément repliée sur un volumen de petit format, sculpté avec un soin poussé jusqu'à faire apparaître le début de sa spire extérieure et la légère déformation que des lectures antérieures lui ont fait subir. Quant à l'homme, il serre dans sa main gauche un codex de quatre tablettes. Le volumen que l'épouse appuie sur l'avant-bras de son mari montre par son petit format qu'il s'agit d'une édition de luxe, d'une taille appréciée des élégantes (de tels livres, peutêtre spécialement réalisés à leur intention, se glissaient facilement dans les plis de leurs vêtements) ${ }^{3}$. La tenue en main d'un volumen de cette sorte montre qu'elle appréciait à tel point la littérature qu'elle se procurait ou se faisait offrir ses auteurs préférés dans un format lui permettant de les avoir partout avec elle, et suggère en outre chez ce couple, qui ne devait pas posséder que ce seul livre, l'existence d'une bibliothèque familiale ${ }^{4}$. 
Enfin, quelques inscriptions gravées dans la pierre contribuent à enrichir nos connaissances, en apportant des témoignages sur une profession qui impliquait la consultation de livres par les femmes qui l'exerçaient : la médecine, comme nous le verrons plus loin.

\section{Catégories et titres de livres associés dans nos sources à un lectorat féminin}

Dans le passage déjà partiellement cité où il évoque un séjour dans une villa proche du Gardon, Sidoine Apollinaire nous apporte une indication d'ordre général sur les livres proposés aux personnes présentes, et parmi elles à leur composante féminine :

"Ils étaient toutefois rangés de telle façon que dans les livres situés près des fauteuils prévus pour les dames on trouvait une inspiration religieuse, alors que ceux qui figuraient du côté des sièges à l'usage des pères de famille se signalaient par le prestige de l'éloquence latine ${ }^{5}$.

Ainsi, dans cette bibliothèque, l'auteur nous montre des mères de famille, des matronae, auxquelles on avait réservé des places pour la lecture, au moyen de confortables fauteuils, alors que leurs époux n'avaient à leur disposition que des sièges sans grand confort, mais plus adaptés à des conversations alimentées par les ouvrages de rhétorique contenus dans les rayonnages dont ils se trouvaient proches. Une telle répartition des livres incitait ces matronae à des lectures religieuses, et tendait simultanément à les dissuader d'aller perturber les conversations des hommes en allant chercher des ouvrages d'un contenu différent dans le secteur attribué à ces derniers.

Le même Sidoine Apollinaire, dans la dernière pièce d'un autre ouvrage, son recueil de poèmes, cite encore une lectrice, en l'occurrence sa cousine Eulalie, et nous fournit une indication sur un des livres destinés à parvenir entre les mains de celle-ci, en l'occurrence ce même recueil : il affecte de s'adresser à cet ouvrage pour lui dire qu'une fois introduit dans la bibliothèque de l'ancien consul Magnus, beau-père de cette cousine, à Narbonne, " ici tu seras lu souvent par ma chère Eulalie " ${ }^{6}$.

Dans un texte remontant à une date antérieure, un autre auteur mettait déjà son livre sous les yeux d'une lectrice de la Gaule, mais sans évoquer cette fois la bibliothèque familiale qui l'accueillait. Il s'agit du poète Martial, qui écrivait durant le dernier tiers du $1^{\text {er }}$ siècle de notre ère. Il le fait en ces termes, dans le genre littéraire qui a créé sa renommée, à savoir une épigramme : "On rapporte que la belle ville de Vienne compte, si la rumeur est vraie, mes petits livres au nombre de ses délices. Chacun m'y lit, vieillard, jeune homme et enfant, ainsi que la vertueuse jeune femme en présence de son sévère mari $"$.

Toutefois, dans ces vers s'observe une contradiction flagrante entre la sévérité du mari et le contenu fréquemment immoral des épigrammes en général et de celles composées par ce poète en particulier, jointe à une évidente exagération dans le nombre de ses lecteurs viennois, présenté comme correspondant à la totalité des habitants de la ville - avec toutefois une nuance d'incrédulité. Ceci nous rappelle que nous nous trouvons en présence d'un genre littéraire à vocation satirique, et qu'il faut voir dans ce passage une moquerie visant ces habitants, et donc un texte à prendre à contrepied pour une partie au moins de son contenu. La vraisemblance porte à n'en retenir que l'existence d'un certain lectorat à Vienne, et à l'intérieur de celui-ci, d'une composante féminine.

\section{Lectures accompagnées de composition littéraire}

Les Gallo-Romaines qui apparaissent dans nos sources ne se bornent pas toujours à une simple lecture. Certaines d'entre elles apparaissent comme se livrant, de manière occasionnelle ou habituelle, à de la composition littéraire. Nous disposons ainsi d'un poème écrit par l'une d'entre elles, demeurée anonyme, en l'occurrence une épitaphe en vers retrouvée à Auch (Eliumberrum), capitale antique de la cité des Auscii, et dédiée à sa chienne Myia (Mouche) ${ }^{8}$ :

"Que celle-ci était douce, qu'elle était aimable, Elle qui durant sa vie se coucha tout contre moi, Partageant toujours mon sommeil et mon lit. Quel malheur, Myia, que tu aies péri.

Tu n'aurais pris la liberté d'aboyer

Que si quelque rival s'était étendu près de ta maîtresse.

Quel malheur, Myia, que tu aies péri.

Un tombeau profond désormais te retient, privée de conscience,

Et tu ne peux ni gronder ni bondir

Ni rayonner de joie en me faisant de douces morsures ". 


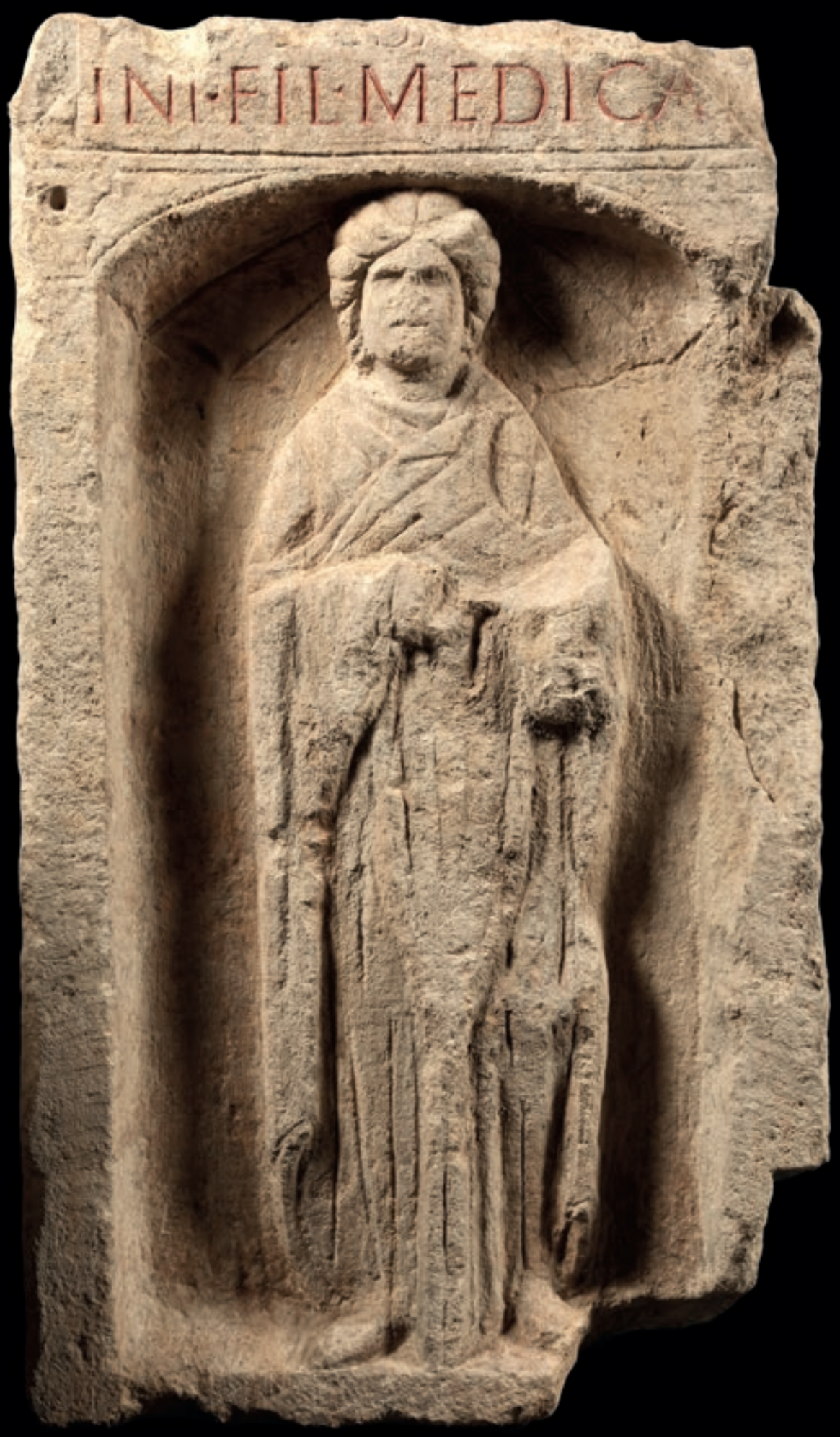

La medica représentée sur une stèle funéraire de Metz (collections Musée de la Cour d'or - Metz Métropole) 


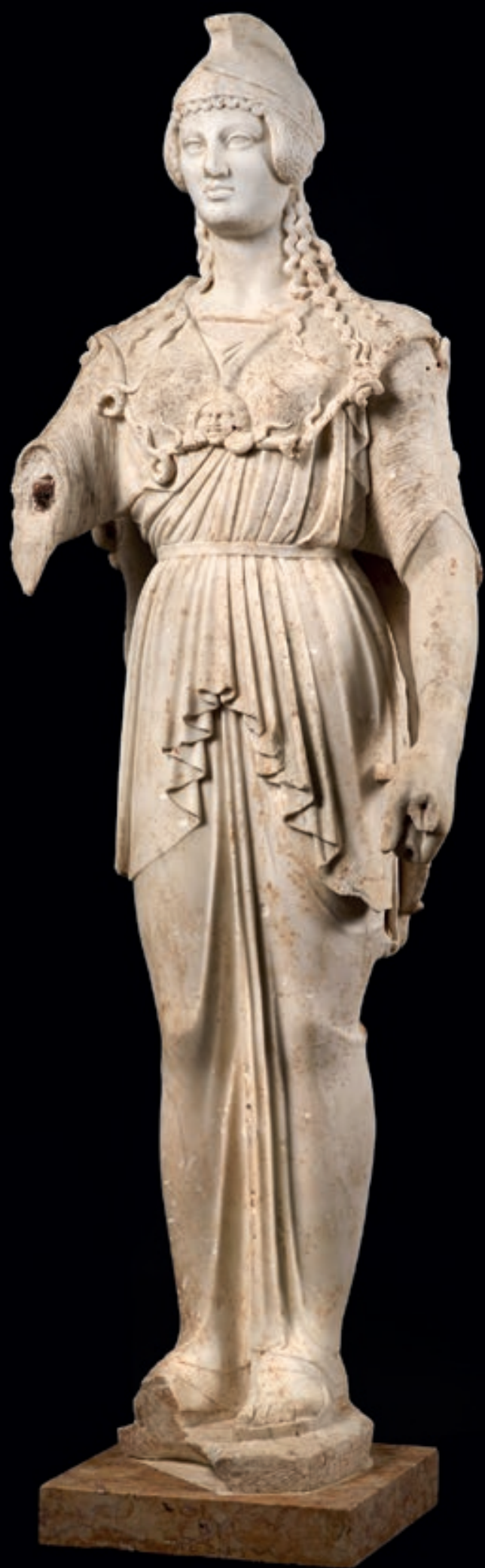

Statue d'Athéna/Minerve découverte dans une domus du centre de Lemonum (Poitiers), et qui pouvait en orner la bibliothèque (coll. Musées de Poitiers) 
Ce petit texte s'inscrit dans une tradition littéraire dont il nous reste une pièce de Catulle dédiée à un moineau défunt et une épigramme de Martial qui fait allusion à cette dernière et décrit une chienne, bien vivante pour sa part ${ }^{9}$. L'habitante d'Auch connaissait sans aucun doute des poèmes relevant de cette tradition. Et pourquoi pas celui de Catulle?

Il est d'autre part arrivé jusqu'à nous une épigramme de trente-deux vers, datée de la fin du $5^{\mathrm{e}}$ siècle et ayant reçu le titre de Vers satiriques contre un certain prétendant. On considère qu'elle a été composée par une habitante de l'Aquitaine, nommée Euchéria ${ }^{10}$. Cette pièce énumère vingt-sept situations impossibles, et sa chute évoque le fait qu'un esclave agricole aux champs oserait solliciter les faveurs de cette Euchéria ${ }^{11}$. En voici les derniers vers :

"Que le triste hibou vive avec la sémillante perdrix, Que la belle colombe niche unie avec le corbeau, Ces alliances monstrueuses changeront à leur profit [les siècles par des destins incestueux. Et ainsi, qu'un esclave des champs recherche donc

[les faveurs d'Euchéria! »"

Dans la mesure où l'on trouve des modèles vraisemblables de ces unions impossibles dans une des Bucoliques de Virgile et dans une Épode d'Horace ${ }^{13}$, et où cette œuvre se caractérise par sa virtuosité, il est permis d'affirmer que cette personne non seulement s'adonnait fréquemment à la composition littéraire en vers, mais aussi qu'elle avait coutume de lire des auteurs classiques.

\section{Lectures professionnelles}

Lorsqu'on pense à l'éventualité d'une utilisation professionnelle des livres par les femmes gallo-romaines, la première idée qui vient à l'esprit est celle d'enseignantes. Quelques-unes se trouvent bien attestées dans le monde romain, mais toutes se localisent hors de Gaule. On ne peut guère envisager de leur rattacher qu'un cas possible, à Bordeaux dans les années 330 de notre ère. Il se trouve présenté en ces termes par saint Jérôme, puis par Prosper d'Aquitaine $^{14}$ : " La fille du rhéteur Nazarius, Eunomia, une jeune chrétienne, égale son père en éloquence ". Sa mention chez ces deux auteurs témoigne du fait qu'elle avait atteint une remarquable célébrité, presque comparable à celle d'Hypatie à Alexandrie, mais ne nous précise pas si elle enseignait la rhétorique, ce qui correspondait à notre actuel niveau universitaire, si elle se produisait devant un public d'amateurs de virtuosité dans l'éloquence, ou encore si elle obtenait un succès exceptionnel dans des exercices d'étudiants, tels que les controverses ou les suasoires. Quoi qu'il en soit, le niveau atteint implique de sa part un recours assidu à une bibliothèque familiale dont l'existence se montrait indispensable à la spécialité du père et de la fille.

Une autre profession qui nécessitait de posséder et de consulter au moins quelques livres se trouve attestée à plusieurs reprises comme ayant été exercée en Gaule par des femmes, en l'occurrence, ainsi qu'il a été annoncé plus haut, la médecine, qui avait inspiré dès l'Antiquité la rédaction de nombreux ouvrages. Une inscription découverte à Lyon et datable du $1^{\text {er }}$ ou du $2^{\text {e }}$ siècle de notre ère signale le financement d'un édifice, dont nous ignorons la nature et la taille, par une femme médecin, une medica, nommée Métilia Donata ${ }^{15}$. Les moyens financiers ayant permis d'offrir ce présent architectural à la ville, sans doute en relation avec une abondante et riche clientèle, suggèrent des compétences élevées qui devaient en partie reposer sur une information d'origine livresque, et donc sur la possession et l'utilisation d'une bibliothèque médicale. Moins parlante, mais également précieuse pour nous, une stèle funéraire découverte à Metz (voir ill. p. 29) porte les restes d'une inscription désignant également comme une medica, en caractères larges et bien détachés, la femme qui s'y trouve représentée en pied ${ }^{16}$, qualification qu'on retrouve également sur l'épitaphe conservée à Nîmes d'une Flavia Hédonè ${ }^{17}$. Outre ces trois medicae, mais cette fois au $4^{\mathrm{e}}$ siècle et à Bordeaux, une tante du poète Ausone, nommée Aemilia Hilaria, et décrite par celui-ci comme pratiquant les arts médicaux ${ }^{18}$, devait également disposer d'une bibliothèque professionnelle, ou bien avoir accès à celle de son beau-frère, le medicus Iulius Ausonius, lui-même père de l'écrivain ${ }^{19}$.

\section{Autre présence féminine : une ou plusieurs déesses}

Une autre présence féminine se remarquait également dans les bibliothèques familiales, mais cette fois sous l'aspect de représentations divines. Il s'agissait de Minerve, et peut-être de Muses. Une statue de la première a été retrouvée dans une domus de Poitiers, l'antique Lemonum, et pourrait y signaler la présence d'une bibliothèque ${ }^{20}$ (voir ill. ci-contre). Plus généralement, une mention 
chez le satiriste Juvénal suggère que de telles figurations se rencontraient communément au milieu des livres ${ }^{21}$. Sidoine Apollinaire comparait sa cousine Eulalie, évoquée plus haut, à Minerve, à cause de sa façon de vivre qui la mettait au rang de cette déesse ${ }^{22}$ : il aurait bien été inspiré par cette présence cultuelle et ornementale.

\section{Présence masculine : accompagnement, surveillance, limitation}

Un contact avec les livres et une fréquentation de bibliothèques familiales ou professionnelles par les femmes se trouvent donc attestés ou suggérés en Gaule romaine. Mais dans une assez forte proportion, nos documents font état d'une présence masculine aux côtés des lectrices : celle de maris ou de membres de la génération précédente, ce qui rappelle qu'elles dépendaient juridiquement d'eux. L'évocation de cette présence prend différentes formes. Elle peut se limiter à la simple mention d'un entourage masculin, dans le cas d'Eulalie, la cousine de Sidoine Apollinaire, lequel écrit, en s'adressant au livre qu'il envoie : " Dirige-toi vers l'ample résidence de l'ancien consul Magnus, et chez ton cher Félix, petit livre. Et dans la bibliothèque qui appartient à son père (...), après t'avoir apprécié, Probus te fera admettre. Ici, tu seras souvent lu par ma chère Eulalie $"{ }^{23}$. Pour sa part, la stèle d'Avaricum montre un mari qui ne se borne pas à une simple présence, mais qui semble reconnu par son épouse comme un guide intellectuel, dans la mesure où elle se trouve figurée derrière lui, appuyant sur son bras une main qui enserre un volumen, et l'autre sur son épaule. Dans le cas de la fille du rhéteur bordelais, celui-ci apparaît comme le modèle qu'elle a égalé.

Mais cette présence se trouve montrée dans certains cas comme limitant l'accès féminin à certaines catégories de livres. Ainsi, le passage de Sidoine Apollinaire sur la bibliothèque de la villa proche du Gardon fait état d'un contrôle masculin des lectures permises aux matronae, facilité par la disposition des lieux et le classement des ouvrages : en l'espèce des sièges spéciaux pour les dames, attractifs par leur confort et disposés près d'armoires exclusivement réservées à la littérature chrétienne. Ce contrôle pourrait même s'être montré quelquefois particulièrement strict, à en croire le qualificatif de tetricus, sévère, attribué au mari de la jeune femme, dans l'épigramme de Martial évoquant son lectorat viennois, et ceci bien qu'il y soit contredit par l'autorisation de lecture accordée à sa jeune épouse.

D'autres documents, à l'inverse, font apparaître des lectrices seules et en situation indépendante. Une stèle funéraire conservée au musée de Saintes montre une femme qui tient de sa main gauche ce qui paraît bien être une enveloppe de cuir et de parchemin serrée par deux cordons, contenant et protégeant un volumen (voir ill. ci-contre). Cette figuration exprimerait une volonté de proclamer qu'elle a durant sa vie accordé une place non négligeable à la lecture ${ }^{24}$. À Auch, l'épitaphe en vers de la chienne Myia suggère fortement à propos de son auteure une absence de mari. Les femmes médecins s'affichent ou se trouvent décrites dans une situation d'indépendance : l'inscription qui mentionne celle de Nîmes ne comporte aucune indication d'ordre familial, non plus que la dédicace de Lyon. La medica de Metz est figurée seule sur sa stèle. Toutefois, dans son cas, sur l'inscription demeurent l'indication " fille de ", plus quelques lettres du nom paternel. Mais une telle indication, en lieu et place de celle d'un mari, pourrait bien exprimer le choix du célibat. Quant à la tante d'Ausone, Aemilia Hilaria, son neveu la décrit en ces termes : " Mais tu offrais sans le cacher l'aspect d'un jeune homme, pratiquant les arts médicaux à la manière des hommes. En toi a toujours existé la haine du sexe féminin, et en conséquence s'est développé l'amour d'une virginité à laquelle tu t'es vouée " ${ }^{25}$, ce qui s'est probablement accompagné d'un refus des situations de dépendance filiale et maritale. Les quatre exemples de medicae cités suggèrent même que l'une des raisons du choix de cette profession, mettant au contact du monde extérieur, aurait été, pour les femmes de la Gaule romaine qui y accédaient, un moyen d'échapper à une condition féminine ressentie comme un enfermement insupportable ${ }^{26}$, sans rompre chaque fois pour autant avec leur famille d'origine.

\section{Que penser de cette présence et de ce contrôle, ou de leur absence?}

Un motif probable de la présence d'un mari ou de parents doit être recherché du côté de la situation des femmes dans la société romaine. Pour elles, une existence normale ne se concevait qu'à l'intérieur d'une famille, représentée à l'extérieur par un élément masculin, père ou époux. L'indication de cette présence était pour elles un gage de respectabilité et de vertu, qualité évoquée à 


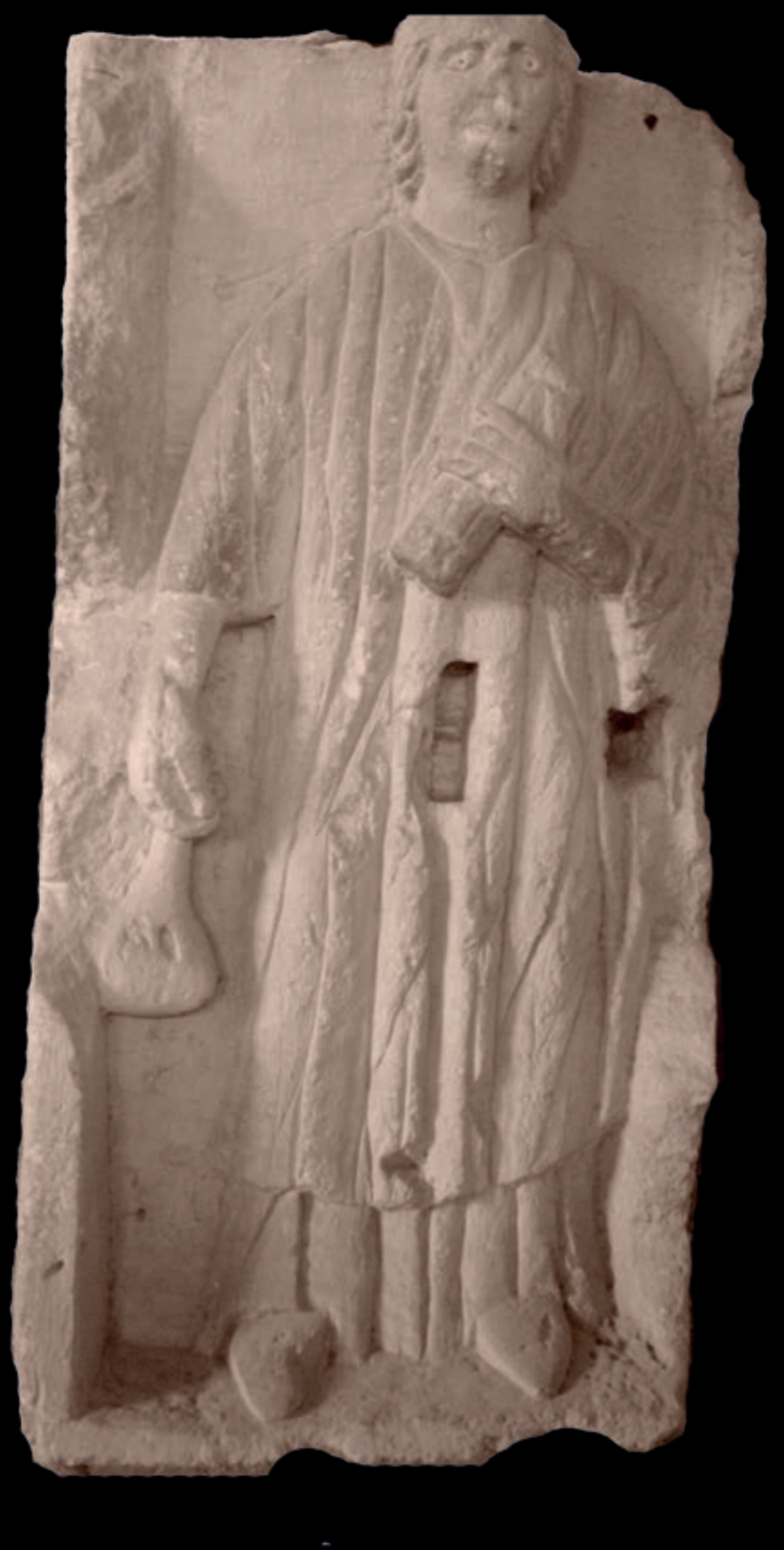
(coll. Musées de la ville de Saintes) 
deux reprises dans nos sources, l'épigramme de Martial qui définit comme casta la jeune Viennoise qu'il présente, d'une manière peu vraisemblable, comme lectrice de son œuvre, ainsi que les vers de Sidoine Apollinaire affirmant que les qualités morales de sa cousine Eulalie la rendent comparable à Minerve et lui valent le respect des austères vieillards et de son beau-père lui-même, ancien consul ${ }^{27}$. La protection de cette vertu et de cette respectabilité explique sans doute le contrôle des lectures et les autorisations limitées d'accès aux bibliothèques familiales évoqués dans nos sources, dans la mesure où certains livres étaient réputés dangereux pour la morale, et les femmes considérées de manière générale comme vulnérables à leur influence, ce qui pouvait comporter un risque pour la stabilité familiale. Mais nous avons vu que la condition familiale faite aux femmes dans la société et les familles gallo-romaines a pu être évitée ou refusée par certaines d'entre elles, comme les vers d'Ausone le laissent penser à propos de sa tante, la medica Aemilia Hilaria, et comme le suggèrent plusieurs documents où un personnage féminin apparaît seul.

Un deuxième motif de la mention d'une présence masculine dans les textes cités semble relever des règles de la bienséance. Elles interdisaient à un homme d'évoquer une femme de la bonne société sans faire simultanément référence à son mari, à son père, ou à la rigueur à un autre proche. Sidoine Apollinaire s'y conforme à propos de sa cousine Eulalie. Il est même possible de dire qu'Ausone, dans le poème qu'il consacre à Aemilia Hilaria, sa tante medica, insère une présence masculine familiale : la sienne propre, puisqu'il s'y présente comme son neveu, et comme le substitut d'un fils.

Peut-être ne s'agit-il donc dans certains de ces textes que d'une indication simplement conventionnelle, d'une rigueur de façade. Qu'en était-il dans les faits ? En quoi consistaient réellement, en l'absence d'invités, les lectures féminines dans la famille propriétaire de la villa du Gardon ? L'époux et le beau-père d'Eulalie, la cousine de Sidoine Apollinaire, contrôlaient-ils ses lectures ou plus probablement s'en remettaient-ils en totalité à des qualités morales qui la rendaient comparable à Minerve aux yeux de son cousin ? Cependant, le fait que Martial ait inclus un sévère mari dans son épigramme nous rappelle qu'il en existait indiscutablement un certain nombre. Mais dans la grande majorité des familles gallo-romaines concernées par cet article, la réalité de l'accès féminin aux livres et aux bibliothèques pourrait bien avoir été, tout compte fait, plutôt permissive et libérale.
En Gaule durant la période romaine, y compris dans ses régions du Nord-Est, et dans les milieux où on leur apprenait à lire, certaines au moins des femmes s'adonnaient à la lecture et avaient recours au contenu des bibliothèques familiales. Mais à en croire nos trop rares sources, l'accès à ces dernières paraît avoir eu le plus souvent lieu en présence du père ou du mari, lesquels pouvaient aller jusqu'à pratiquer un contrôle strict et une limitation de l'accès aux livres, pour des raisons essentiellement morales. En fait, plusieurs d'entre les Gallo-Romaines semblent y avoir échappé et s'être assuré une indépendance dans ce domaine, notamment créée par des choix professionnels où l'exercice de la médecine aurait tenu une place non négligeable. D'autre part, les affirmations textuelles et iconographiques de cette présence, de ce contrôle et de cette limitation ne doivent peut-être pas être trop être prises au pied de la lettre, sauf exception : elles pourraient en partie avoir répondu à des conventions sociales et mondaines plutôt qu'exprimer une pratique réelle, et la situation vécue devait se montrer beaucoup plus variée. Quoi qu'il en soit, l'accès des femmes aux livres et aux bibliothèques, dans les familles qui en possédaient, même contrôlé et limité, est à considérer comme un aspect non négligeable de la civilisation gallo-romaine. Elles participaient de la sorte par la lecture, mais parfois aussi par la composition littéraire, à l'activité culturelle de leur famille et, pardelà, à celle de la Gaule.

\section{Robert Bedon}

\section{Notes}

1- Sidoine Apollinaire, Lettres, II, 9, 4: Huc libri adfatim in promptu. Videre tu crederes aut grammaticales pluteos aut Athenaei cuneos aut armaria exstructa bybliopolarum. Sic tamen quod, qui inter matronarum cathedras codices erant... L’Athénée était une sorte d'université créée à Rome par l'empereur Hadrien.

2 - Collections des Musées de la ville de Bourges. Musée du Berry. Inv. 915.5.3, $\mathrm{n}^{\circ}$ 12. P. Bailly, "Visages des habitants d'Avaricum ", in Archéologia, 132, juillet 1979, p. 38-39, photo 7. J.-Fr. Chevrot, J. Troadec, Carte archéologique de la Gaule, Le Cher, 18, Paris, 1992, p. 141, nº 605.

3 - Entre autres auteurs, W. Schubart, Das Buch bei den Griechen und Römern. Eine Studie aus der Berliner Papyrussammlung, Berlin, 1921, p. 57-58.

4 - D'autres reliefs funéraires pourraient montrer aussi une pratique féminine de la lecture, comme une stèle de Monthureux (dép ${ }^{t}$. des Vosges), qui figure un couple dont l'épouse porte, suspendu au poignet, un étui susceptible de contenir un volumen ou plus probablement des stylets ou des calames : G. Moitrieux, J.-N. Castorrio (collab.), Toul et la cité des Leuques, tome III du Recueil général des sculptures sur pierre de la Gaule, H. Lavagne éd., Paris, 2010, $\mathrm{n}^{\circ} 376$ et pl. 75 .

5 - Sic tamen quod, qui inter matronarum cathedras codices erant, stilus his religiosus inueniebatur, qui uero per subsellia patrumfamilias, hi cothurno Latiaris eloquii nobilitabantur. 
6 - Sidoine Apollinaire, Poèmes, XXIV, vers 95 : Hic saepe Eulaliae meae legeris.

7- Martial, Epigrammes, VII, 88, vers 1-4 : Fertur habere meos, si uera est fama, libellos / Inter delicias pulchra Vienna suas:/ Me legit omnis ibi senior iuuenisque puerque, / Et coram tetrico casta puella uiro.

8 - Corpus Inscriptionum Latinarum (CIL), XIII, 488 : Quam dulcis fuit ista, quam benigna, / Quae cum uiueret in sinu iacebat, / Somni conscia semper et cubilis. I O factum male Myia quod peristi./ Latrares modo si quis adcumberet / Riualis dominae licentiosa. / O factum male Myia quod peristi./ Altum iam tenet insciam sepulcrum, / Nec s(a)euire potes nec insilire, / Nec blandis mihi morsib(us) renides. Voir G. Achard, "Poésie et rhétorique dans les inscriptions antiques de la Gaule ", in La langue des inscriptions latines de la Gaule, Lyon, 1989, p. $117-133$ (p. 125 et 127), qui situe cette Auscitaine dans « le milieu culturel supérieur" ".

9 - Catulle ( $1^{\text {er }}$ siècle av. J.-C.), Poèmes, 2. Martial, Ép., I, vers 109.

10- Elle aurait appartenu à la famille d'Eucher, évêque de Lyon.

11 - S. Santelia, Per amare Eucheria, Bari, éd. Palomar, 2005

12- Euchéria, Satirici versus in quemdam procum, vers 29-32 :

Tristis perspicua sit cum perdice cauannus,

Iunctaque cum coruo pulchra columba cubet,

Haec monstra incertis mutent sibi tempora fatis.

Rusticus et seruus sic petat Eucheriam.

13 - Virgile, Bucoliques, VIII, vers 26-32. Horace, Épodes, XVI, vers 25-34.

14- Jérôme, Chronique, p. 233, 1 (année 236); Prosper d'Aquitaine, Chronique, dans J.-P. Migne, Patrologie latine, tome 51, Paris, 1861, col. 576 : Nazarii rhetoris filia Eunomia christiana uirgo in eloquentia patri coaequatur. Un rhéteur était un professeur d'enseignement supérieur. Nazarius se trouve localisé à Bordeaux par Ausone, in Commémoration des professeurs bordelais, XIV, vers 9-12.

15- CIL, XIII, 2019. N. Mathieu et B. Rémy, Les femmes en Gaule romaine ( ${ }^{\text {er }}$ siècle av.J.-C. - V siècle apr.J.-C.), Paris, 2009, p. 106

16 - CIL, XIII, 4334 et E. Espérandieu, Recueil général des bas-reliefs de la Gaule romaine, V, Paris, 1913, $\mathrm{n}^{\circ}$ 4363. L. Ayache, K. Kazek, « Les Gallo-Romains vus par eux-mêmes ", in L'Archéologue, 119, avril-mai 2012, p. 19

17 - CIL, XII, 3343, N. Mathieu et B. Rémy, op. cit., p. 106 : Flauiae/Hedones/medicae/ ex testa[mento]

18- Ausone, Parentalia, 6, vers $6:$ medicis artibus experiens

19- Ausone, Parentalia, 1

20-G.-Ch. Picard, "L'influence néo-attique dans la Gaule de l'Ouest ", in Comptes rendus de l'Académie des Inscriptions et Belles-Lettres, 1984, p. 242-249 (p. 242-243). J.-Ch. Balty, "Statuaire idéale et romanisation en Narbonnaise et dans les Trois Gaules ", in Bulletin de la Société Française d'Archéologie Classique, XXXV, 2002-2003, p. 168-170 (p. 169-170).

21 - Juvénal, Satires, III, vers 219

22- Sidoine Apollinaire, Poèmes, XXIV, vers 96-98: "Cuius Cecropiae pares Mineruae / mores et rigidi senes et ipse / quondam purpureus socer timebant, toi dont à la fois les austères vieillards et ton beau-père lui-même, ancien consul, respectaient la façon de vivre qui la mettait au rang de la Minerve Cécropia ". Le qualificatif de Cecropia, signifiant Patronne d'Athènes, est une référence à Cécrops, le premier roi de cette cité.

23- Sidoine Apollinaire, Poèmes, XXIV, vers 90-95: Hinc ad consulis ampla tecta Magni / Felicemque tuum ueni, libelle. / Et te bybliotheca qua paterna est (...)/ Admittifaciet Probus probatum. / Hic saepe Eulaliae meae legeris.

24-Au Musée archéologique de la ville. Inv. 1949.476A. E. Espérandieu, Recueil..., II, Paris, $1908, \mathrm{n}^{\circ}$ 1350. Sur cette protection des volumina : Lygdamus, Élégies, I, vers 9, et surtout Catulle, Poèmes, XXII, vers 7.

25- Ausone, Parentalia, VI, vers 5-8:

Reddebas uerum non dissimulantem ephebum,

More uirum medicis artibus experiens,

Femini sexus odium tibi semper et inde

Creuit deuotae uirginitatis amor.

26-Entre autres, N. Mathieu et B. Rémy, op. cit., p. 106

27 - Sidoine Apollinaire, Poèmes, XXIV, vers 96-98 : voir note 22

28- A. Provost, « La villa maritime de Mané-Véchen à Plouhinec (Morbihan) ", in Aremorica, 1,2007 , p. 85-100

\section{Un apport fourni par l'archéologie des bâtiments?}

A-t-on retrouvé en Gaule des vestiges provenant de ces bibliothèques familiales dont les patresfamilias permettaient l'accès à leurs mères, à leurs épouses et à leurs filles aux diverses conditions que nous avons rencontrées ? Une luxueuse villa fouillée en Armorique, à Mané-Véchen, sur la commune de Plouhinec (dépt. du Morbihan), près de la rivière d'Étel ${ }^{28}$, possédait une petite salle (3,75 sur 2,88 $\mathrm{m}$.) dont l'architecture donne à penser qu'elle pourrait bien avoir été conçue pour cet usage (voir ill. ci-dessous). Elle présente en effet dans ses murs des renfoncements qui ont probablement contenu des armoires ou des rayonnages destinés à recevoir des livres, comme il s'en rencontre dans les bibliothèques publiques du monde romain. Mais une grande partie des collections de livres présentes dans certaines des familles de la Gaule romaine devait simplement prendre place dans des boîtes ou des coffres, ou encore dans une ou dans quelques armoires, à l'intérieur de pièces dépourvues de particularités dans leur structure, et il n'en demeure pas de traces.

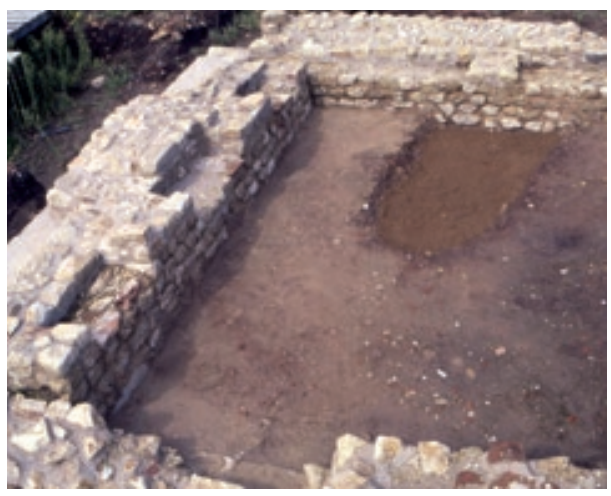

Petite salle appartenant à la villa de Mané-Véchen et interprétable comme conçue pour un usage de bibliothèque, à cause des renfoncements ménagés dans ses murs. 